\title{
Nonlinear quantum capacitance
}

\author{
Baigeng Wang, Xuean Zhao, and Jian Wang \\ Department of Physics, The University of Hong Kong, Pokfulam Road, Hong Kong, China \\ Hong Guo a) \\ Center for the Physics of Materials and Department of Physics, McGill University, Montreal, \\ Quebec, H3A 2T8 Canada
}

(Received 1 February 1999; accepted for publication 14 March 1999)

\begin{abstract}
We analyze the nonlinear voltage dependence of electrochemical capacitance for nanoscale conductors. This voltage dependence is due to the finite density of states of the conductors. Within Hartree theory we derive an exact expression for the electrochemical capacitance-voltage curve for a parallel plate system. The result suggests a quantum scanning capacitance microscopy at the nanoscale: by inverting the capacitance-voltage expression one is able to deduce the local spectral function of the nanoscale conductor. () 1999 American Institute of Physics.
\end{abstract}

[S0003-6951(99)04319-3]

It has been well known that density of states affects the capacitance $^{1}$ of a system. In this work we investigate a nonlinear bias voltage dependence of the electrochemical capacitance of a model capacitor at the nanoscale for which the density of states (DOS) also plays the crucial role. The physical origin of this bias dependence which we have examined is not because of depletion of charges on a capacitor plate, which has been understood in semiconductor research, but because of the finite DOS of the plates. We show that the accumulated charge on a conductor has a nonlinear bias dependence due to DOS effects, and it is this nonlinear charge that leads to the nonlinear capacitance. For conductors at nanoscale, it is known ${ }^{2}$ that the very small DOS plays an important role in the behavior of the capacitance. However, the DOS induced nonlinear capacitance has not been studied ${ }^{3}$ before and our investigation suggests an interesting application that is the quantum scanning capacitance microscopy.

Central to the problem is the determination of charge $\rho_{\alpha}$ accumulated on a conductor labeled by $\alpha$. In general $\rho_{\alpha}$ is a nonlinear function of bias voltages $\left\{V_{\beta}\right\}$

$$
\begin{aligned}
\rho_{\alpha} & =\sum_{\beta} C_{\alpha \beta} V_{\beta}+\frac{1}{2} \sum_{\beta \gamma} C_{\alpha \beta \gamma} V_{\beta} V_{\gamma}+\ldots \\
& \equiv \sum_{\beta} C_{\alpha \beta}\left(\left\{V_{\gamma}\right\}\right) V_{\beta},
\end{aligned}
$$

where $C_{\alpha \beta}=\partial_{V_{\beta}} \rho_{\alpha}$ is the usual electrochemical capacitance, ${ }^{2,4} C_{\alpha \beta \gamma}=\partial_{V_{\beta}} \partial_{V_{\gamma}} \rho_{\alpha},{ }^{5}$ etc., are the nonlinear electrochemical capacitance coefficients, and $C_{\alpha \beta}\left(\left\{V_{\alpha}\right\}\right)$ is the general voltage dependent nonlinear capacitance. So far investigations on quantum correction to capacitance ${ }^{1,2,4}$ have only considered the linear term, but in this work we focus on a general nonlinear expression for $C_{\alpha \beta}\left(\left\{V_{\gamma}\right\}\right)$.

To be specific we consider a model parallel plate capacitor connected to electron reservoirs by perfect leads, ${ }^{2}$ schematically shown in the inset of the Fig. 1. We adopt the dynamic point of view ${ }^{6}$ to calculate the electrochemical ca-

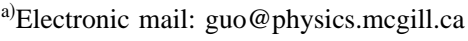

pacitance, hence a finite bias applied at the reservoirs injects a charge density into the capacitor plates which, through interaction, induces a local response. The total net charge (the sum of injected and induced charge) at a plate $\alpha, \rho_{\alpha}$, is thus established at equilibrium. We calculate $\rho_{\alpha}$ by extending the standard nonequilibrium Green's function (NEGF) technique. To save space we refer interested readers to literature for standard technical details; here we will only discuss the most important extension to the standard approach.

The quantum scattering (injection of electrons) is determined by the retarded and advanced Green's function $G^{r, a}(E, U)$ : note that we have explicitly included the electrostatic potential buildup inside our capacitor, $U=U(\mathbf{r})$, into these Green's functions. In the Hartree approximation ${ }^{7}$

$$
G^{r, a}(E, U)=\frac{1}{E-H-q U-\Sigma^{r, a}},
$$

where $H$ is the Hamiltonian for our nanoscale conductors

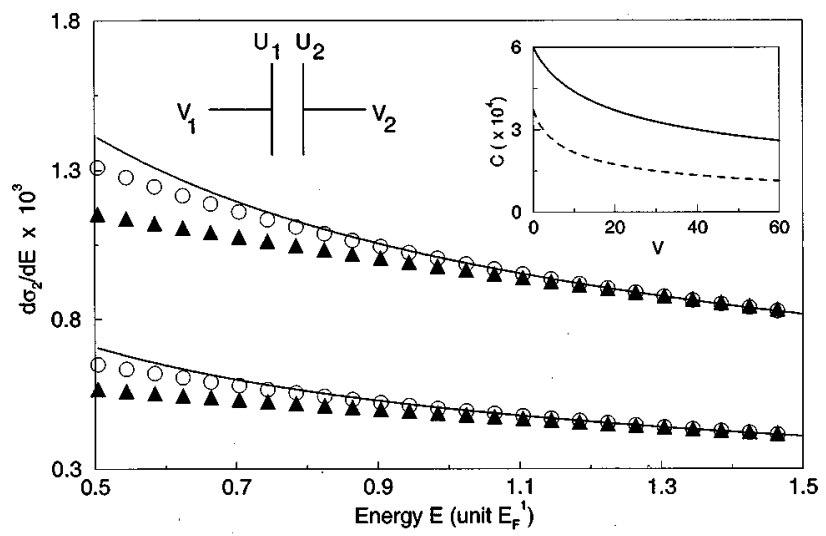

FIG. 1. Operation of QSCM: comparison of "measured" LPDOS to the exact one. (Solid lines) exact; (circles) fitted LPDOS using ten voltages; (triangles) fitted LPDOS using three voltages. Upper curves are for $b_{2}$ $=0.002$, lower curves for $b_{2}=0.001$. The QSCM tip has been fixed with $b_{1}=0.003$. Upper right inset: the electrochemical capacitance vs voltage $V\left(V=V_{2}-V_{1}\right)$ for the two sets of $b_{2}$ : upper curve is for $b_{2}=0.002$. The unit of $V$ is $E_{F}^{1} / e$. Upper left inset: schematic plot of the parallel plate mesoscopic capacitor with $V_{1,2}$ the external bias and $U_{1,2}$ the internal potential. 
written in the familiar second quantized form, ${ }^{8} \Sigma^{r, a}$ is the self-energy describing the coupling between the conductors to the leads which is calculated in standard fashion. ${ }^{9,10} \mathrm{At}$ Hartree level we determine $U(\mathbf{r})$ by the self-consistent Poisson equation

$$
\nabla^{2} U=4 \pi i q \int(d E / 2 \pi) G^{<}(E, U),
$$

where the right hand side is just the total net charge distribution in our conductors. Within Hartree approximation

$$
G^{<}(E, U)=G^{r} \sum_{\beta} i \Gamma_{\beta}\left(E-q V_{\beta}\right) f\left(E-q V_{\beta}\right) G^{a},
$$

where $\Gamma_{\beta}$ is the voltage dependent coupling parameter between probe $\beta$ and the scattering region., ${ }^{9,10}$ The selfconsistent equations (2), (3), and (4) completely determine the nonlinear physics at the Hartree level. We emphasize again that the important departure of our theory from the familiar NEGF analysis ${ }^{9-11}$ is that we explicitly include the internal potential landscape $U(\mathbf{r})$ into the Green's functions self-consistently.

The net charge pileup on a capacitor plate, measured from the equilibrium background, is derived from the right hand side of Eq. (3). In the wideband $\operatorname{limit}^{12}\left(\Gamma_{\alpha}\right.$ $=$ constant)

$$
\rho_{\alpha}(x)=\int \frac{d E}{2 \pi} \Gamma_{\alpha}\left[G^{r} G^{a} f\left(E-q V_{\alpha}\right)-G_{0}^{r} G_{0}^{a} f(E)\right]_{x x},
$$

where $G_{0}^{r, a}$ are the equilibrium Green's functions. Next, we formally expand $G^{r}$ and $G^{a}$ in a power series of the internal potential $U$, and expand the Fermi function in series of the bias voltage $V_{\alpha}$. Collecting terms according to the powers of $U$ and $V_{\alpha}$, Eq. (5) reduces to the following infinite series which can be exactly summed:

$$
\begin{aligned}
\rho_{\alpha}(x) & =\left[\frac{d \sigma_{\alpha}}{d E}\left(V_{\alpha}-U\right)+\frac{1}{2} \frac{d^{2} \sigma_{\alpha}}{d E^{2}}\left(V_{\alpha}-U\right)^{2}+\cdots\right]_{x x} \\
& \equiv \sigma_{\alpha}\left(E+V_{\alpha}-U\right)-\sigma_{\alpha}(E),
\end{aligned}
$$

where the quantity $d \sigma_{\alpha} / d E$ is defined as

$$
\frac{d \sigma_{\alpha}}{d E} \equiv-\int \frac{d E}{2 \pi}\left(G_{0}^{r} \Gamma_{\alpha} \frac{\partial f}{\partial E} G_{0}^{a}\right)_{x x} .
$$

The physical significance of the quantity $d \sigma_{\alpha} / d E$ can be identified as the linear spatial dependent local partial density of states (LPDOS). ${ }^{2,5}$ Expression (7) has been obtained before. ${ }^{13,5}$ For a conductor, which is weakly coupled to external leads, LPDOS gives the local DOS of this conductor. Hence the spectral function $\sigma_{\alpha}(E)$ characterizes the local electronic structure of a nanoscale conductor.

The nonlinear charge distribution gives the general electrochemical capacitance versus voltage curve

$$
C=\left[\sigma_{1}\left(E+V_{1}-U_{1}\right)-\sigma_{1}(E)\right] /\left(V_{1}-V_{2}\right),
$$

where we have set electron charge $q$ to be unity. To determine $C$ we must obtain internal potentials $U_{1}$ and $U_{2}$ at the two plates. For this purpose we introduce the geometrical capacitance ${ }^{6} C_{0}$

Downloaded 14 Nov 2006 to 147.8.21.97. Redistribution subject to AIP license or copyright, see http://apl.aip.org/apl/copyright.jsp and

$$
C_{0}=-\left[\sigma_{2}\left(E+V_{2}-U_{2}\right)-\sigma_{2}(E)\right] /\left(U_{1}-U_{2}\right) .
$$

For a parallel plate capacitor $C_{0}=A /(4 \pi a)$, where $A$ is the area of the plates and $a$ is their separation. In general $C_{0}$ can be calculated numerically. The two equations (9) and (10) determine the internal potentials $U_{1}$ and $U_{2}$ when the scattering spectral function $\sigma_{\alpha}$ and $C_{0}$ are known. Equations (8), (9), and (10) give the general electrochemical capacitancevoltage $(C-V)$ curve for quantum capacitors. It is universal in the sense that system specific parameters only appear in the scattering spectral functions of the conductors. From these general results, two useful applications follow:

$C-V$ curve. Our general results allow us to predict $C-V$ curves. For this application we note that there are well established methods for calculating the scattering LPDOS: ${ }^{14,5}$ either by Eq. (7) after evaluating the Green's functions, or using the scattering wave functions ${ }^{14} \psi, \quad d \sigma_{\alpha} / d E$ $=|\psi|^{2} / h v$, where $v \sim \sqrt{E}$ is the velocity and $h$ is the Planck constant. Hence by solving a quantum scattering problem one obtains $\sigma_{\alpha}$. Let us consider a case where $\psi$ is not very sensitive to $E$, thus $d \sigma_{\alpha}(E) / d E \approx b_{\alpha} /(2 \sqrt{E})$ or $\sigma_{\alpha}(E)$ $=b_{\alpha} \sqrt{E}$, where $b_{\alpha}$ is a constant. For this LPDOS, solving Eqs. (8), (9), and (10) we obtain

$$
C\left(V_{1}-V_{2}\right)=\frac{\beta-\sqrt{\beta^{2}-4\left(b_{1}^{2}-b_{2}^{2}\right) b_{1}^{2} b_{2}^{2}\left(V_{1}-V_{2}\right)}}{2\left(b_{1}^{2}-b_{2}^{2}\right)\left(V_{1}-V_{2}\right)},
$$

where

$$
\beta=4 \pi a b_{1}^{2} b_{2}^{2}+2 b_{1} b_{2}\left(b_{1}+b_{2}\right) \sqrt{E} .
$$

The inset of Fig. 1 shows this electrochemical $C-V$ curve as a function of $V \equiv\left(V_{2}-V_{1}\right)$ for two sets of parameters $b_{\alpha}$. The physical reason that $C$ changes with $V$ is because the plates have finite DOS. Indeed, by making DOS very large $\left(b_{1}, b_{2} \rightarrow \infty\right)$ the voltage dependence of Eq. (11) disappears and $C$ becomes purely geometrical. Furthermore, it can be confirmed that formula (11) recovers the linear ${ }^{2}$ and second order nonlinear ${ }^{5}$ capacitance coefficients when we take the $\left(V_{2}-V_{1}\right) \rightarrow 0$ limit.

Quantum scanning capacitance microscopy. Our general results suggest a quantum scanning capacitance microscopy (QSCM). This idea naturally follows from the results presented above: since the electrochemical capacitance varies with bias due to a finite DOS of the conductors involved, we should be able to find the DOS by measuring $C$. Essentially we wish to obtain spectral function $\sigma_{2}(E)$ or local density of states $d \sigma_{2} / d E$ as a function of energy for an unknown conductor, from a known $\sigma_{1}(E)$ of our QSCM "tip" which has been calibrated. ${ }^{15}$ As the QSCM tip is scanned along the surface of a nano-scale conductor, or along the surface of a planar dielectric layer with nanoconductors buried underneath, experimentally one can measure the $C(V)$ curves at each spatial position.

From Eq. (8), we obtain $U_{1}$ as a function of potential difference $V$ using the known $\sigma_{1}$ and the measured $C(V)$, by solving the equation $\sigma_{1}\left(1-U_{1}\right)-\sigma_{1}(1)=V C(V)$, where we have set $V_{1}=0, V_{2}=V$, and $E=1$ is set at the Fermi

$$
C_{0}=\left[\sigma_{1}\left(E+V_{1}-U_{1}\right)-\sigma_{1}(E)\right] /\left(U_{1}-U_{2}\right),
$$


energy of the QSCM tip [ $C(V)$ is measured at Fermi energy of the tip $E_{F}^{1}$, e.g., Eq. (11)]. Next, From Eq. (9) we obtain $U_{2}(V)$. With $U_{1}$ and $U_{2}$ we finally find $\sigma_{2}(E)$ from Eq. (10). In particular we can solve the spectral function $\sigma_{2}$ by representing it into a polynomial:

$$
\sigma_{2}(X)=\sum_{m=0}^{n} y_{m} X^{m}
$$

where the coefficients $y_{m} \equiv\left(d^{m} \sigma_{2} / d X^{m}\right) /(m !)$ are just the linear $(m=1)$ LPDOS and nonlinear $(m>1)$ LPDOS. $^{5}$ They are obtained by solving the following set of linear algebraic equations which come from Eq. (10):

$U_{2}\left(V_{j}\right)-U_{1}\left(V_{j}\right)=4 \pi a \sum_{m=0}^{n} y_{m}\left\{\left[1+V_{j}-U_{2}\left(V_{j}\right)\right]^{m}-1\right\}$,

where $j=1,2, \cdots, n$. Hence by making experimental measurements at $n$ different voltages $V_{j}$, we obtain the functional form of $\sigma_{2}(E)$ from Eq. (13). Figure 1 demonstrates the principle of QSCM. We use Eq. (11) as the experimentally measured $C-V$ curve (the inset) to simulate a measurement. Then using the QSCM "measured" $\sigma_{2}(E)$ from Eq. (13), we plot the local density of states $d \sigma_{2} / d E$ versus energy $E$. The solid line is the exact $d \sigma_{2} / d E=b_{2} /(2 \sqrt{E})$ and the dots are the QSCM result. We used ten voltages in solving Eq. (14) and the outcome is quite good, while using three voltages it already represents a rough trend.

In summary, we have developed a general nonlinear dc theory which is applied to investigate the full nonlinear charge distribution in nanoscale conductors. Within the Hartree theory we derived an exact expression of the electrochemical capacitance versus external bias voltage curve for quantum capacitors. This result is generic in the sense that all system specific information is included in the scattering local density of states. Hence the $C-V$ formula has a wide range of applicability. By inverting this formula, we propose a novel QSCM. The QSCM extends the ability of the usual scanning capacitance microscopy: ${ }^{16}$ QSCM includes the quantum corrections to capacitance in mapping out the spatial charge distribution; and it gives the local density of states as a function of electron energy.

One of the authors, J.W., is supported by a RGC grant from the SAR Government of Hong Kong under Grant No. HKU 7112/97P, and a CRCG grant from the University of Hong Kong. H.G is supported by NSERC of Canada and FCAR of Québec.

${ }^{1}$ T. P. Smith, B. B. Goldberg, P. J. Stiles, and M. Heiblum, Phys. Rev. B 32, 2696 (1985).

${ }^{2}$ M. Büttiker, J. Phys.: Condens. Matter 5, 9361 (1993).

${ }^{3}$ Recently Blanter and Büttiker discussed the nonlinear charge effects and nonlinear capacitance as related to shot noise in resonant quantum wells. Ya. M. Blanter and M. Büttiker, Phys. Rev. B 59, 10217 (1999).

${ }^{4}$ J. Wang, H. Guo, J-L. Mozos, C. C. Wan, G. Taraschi, and Q. R. Zheng, Phys. Rev. Lett. 80, 4277 (1998).

${ }^{5}$ Z. S. Ma, J. Wang, and H. Guo, Phys. Rev. B. 59, 7575 (1999).

${ }^{6}$ T. Christen and M. Büttiker, Phys. Rev. Lett. 77, 143 (1996).

${ }^{7}$ S. Datta, Electronic Transport in Mesoscopic Systems (Cambridge University Press, New York 1995).

${ }^{8}$ B. Wang, J. Wang, and H. Guo, Phys. Rev. Lett. 82, 398 (1999).

${ }^{9}$ A. P. Jauho, N. S. Wingreen, and Y. Meir, Phys. Rev. B 50, 5528 (1994).

${ }^{10}$ M. P. Anantram and S. Datta, Phys. Rev. B 51, 7632 (1995).

${ }^{11}$ The current-voltage $(I-V)$ curve of a tunneling system within NEGF formalism has been studied by including the internal potential using a linear capacitive charging model. See C. A. Stafford, Phys. Rev. Lett. 77, 2770 (1996).

${ }^{12}$ Y. Meir, N. S. Wingreen, and P. A. Lee, Phys. Rev. Lett. 70, 2601 (1993); T. K. Ng, ibid. 70, 3635 (1993); C. Bruder and H. Schoeller, ibid. 72, 1076 (1994); S. König, H. Schoeller, and G. Schön, ibid. 76, 1715 (1996).

${ }^{13}$ T. Gramespacher and M. Büttiker, Phys. Rev. B 56, 13026 (1997).

${ }^{14} \mathrm{M}$. Büttiker and T. Christen, in Quantum Transport in Semiconductor Submicron Structures, edited by B. Kramer (Kluwer Academic, Dordrecht, 1996), p. 263.

${ }^{15}$ The calibration can be done in several ways. For example one can prepare two identical conductors thus $\sigma_{1}(E)=\sigma_{2}(E)$, and solve for $\sigma_{1}$.

${ }^{16}$ In conventional SCM a classical geometrical capacitance is assumed. See, for example, A. N. Erickson, D. M. Adderton, Y. E. Strausser, and R. J. Tench, Semicond. Sci. Technol. June, 125 (1997); J. Electron. Mater. 25, 301 (1996). 